\title{
RING1 and YY1 binding protein suppresses breast cancer growth and metastasis
}

\author{
HONGYAN ZHOU ${ }^{1 *}$, JIE LI $^{2 *}$, ZHANQIANG ZHANG ${ }^{2 *}$, RUNYI YE ${ }^{2}$, \\ NAN SHAO ${ }^{2}$, TUCKYUN CHEANG ${ }^{2,3}$ and SHENMING WANG ${ }^{2}$
}

\begin{abstract}
Departments of ${ }^{1}$ Neurological Intensive Care Unit, ${ }^{2}$ Breast and Thyroid Surgery, and ${ }^{3}$ Laboratory of General Surgery, The First Affiliated Hospital of Sun Yat-sen University, Guangzhou, Guangdong 510080, P.R. China
\end{abstract}

Received June 12, 2016; Accepted September 16, 2016

DOI: $10.3892 /$ ijo. 2016.3718

\begin{abstract}
Evidence suggests that RING1 and YY1 binding protein (RYBP) functions as a tumor suppressor. However, its role in breast cancer remains unclear. In the present study, the expression of RYBP was assessed in breast cancer patients and cell lines. Disease-free survival durations of breast cancer patients with high RYBP expression were determined based on the ATCG dataset. The effects of RYBP overexpression on cell growth, migration and invasive potency were also assessed. Nude mouse xenograft and lung metastasis models were also used to confirm the role of RYBP. The involvement of SRRM3 in RYBP-mediated breast cancer suppression was explored using SRRM3 siRNA. The potential relationship between RYBP, SRRM3, and REST-003 was examined by qPCR. The results showed that RYBP was downregulated in breast cancer patients and in several breast cancer cell lines. Breast cancer patients with high expression levels of RYBP displayed better disease-free survival. Overexpression of RYBP in MDA-MB-231 and SK-BR-3 cells significantly decreased cell proliferation, migration, and invasion ability, and increased the proportion of cells arrested in S-phase compared with the negative control cells. Additionally, upregulation of proliferation-related cell cycle proteins (cyclin A and cyclin B1) and E-cadherin, and downregulation of snail were observed in
\end{abstract}

Correspondence to: Dr Tuckyun Cheang or Dr Shenming Wang, Department of Breast and Thyroid Surgery, The First Affiliated Hospital of Sun Yat-sen University, Guangzhou, Guangdong 510080, P.R. China

E-mail: zhangdeyuansci@163.com

E-mail: shenmingw6@163.com

*Contributed equally

Abbreviations: RYBP, RING1 and YY1 binding protein; PcG, polycomb; NSCLC: non-small cell lung cancer; HCC, hepatocellular carcinoma; DFS, disease-free survival; TCGA: The Cancer Genome Atlas; EMT, epithelial-mesenchymal transition; SRRM3, serine/arginine repeat-related protein; REST, RE1-silencing transcription

Key words: RYBP, breast cancer, metastasis, SRRM3, REST-003
RYBP-overexpressing cells. Overexpression of RYBP reduced tumor volume and weight as well as metastatic foci in the lungs of nude mice. SRRM3 knockdown by siRNA, which is downregulated after RYBP overexpression, suppressed cell growth and metastasis in MDA-MB-231 and SK-BR-3 cells. Furthermore, qPCR analysis revealed that REST-003 ncRNA was downregulated in cells overexpressing RYBP and in SRRM3-inhibited cells. Moreover, cell invasion ability and growth were increased after SRRM3 upregulation in RYBP-overexpressing cells, but they were decreased following si-REST-003 transfection. In conclusion, overexpression of RYBP suppresses breast cancer growth and metastasis both in vitro and in vivo. SRRM3 and REST-003, which are downregulated in cells overexpressing RYBP, may be involved in RYBP-mediated breast cancer progression.

\section{Introduction}

Breast cancer is the most common form of cancer in women. According to the World Cancer Report, there are more than 1.6 million new cases annually in women (1). In China, breast cancer was estimated to account for $15 \%$ of all newly diagnosed cancers in women in 2015 and is thought to be the leading cause of cancer death in women under the age of 45 years (2). Although the overall mortality rate has declined due to the greater efficacy of therapeutic interventions, recurrence and metastasis are still major challenges in tackling the disease (3). Therefore, investigations of progression-related molecular mechanisms will significantly benefit targeted breast cancer therapy and improve disease outcomes.

RYBP (RING1- and YY1-binding protein) has been shown to interact with RING1, RNF2 and YY1 (4), and is a member of the polycomb ( $\mathrm{PcG}$ ) group of proteins (5). It has been shown to be downregulated in several types of tumors (6-8). Overexpression of RYBP using an adenovirus-based system has been shown to decrease cell proliferation in various cancer cell lines, including U2OS, SAOS2, A549, TOV112 and TOV21 (9). In a study of integrative genomic profiling of human prostate cancer, RYBP was implicated as a potential cooperative tumor suppressor (10). Regarding its role in prognosis, an integrative analysis of gene expression in cervical cancer indicated that patients with low expression levels of RYBP have shorter progression-free survival rates (11). Recently, downregulation 
of RYBP has been found to decrease cell sensitivity to chemotherapy, and is associated with poor prognosis in patients with non-small cell lung cancer (NSCLC) and hepatocellular carcinoma (HCC) $(8,12)$. However, whether RYBP plays a role in breast cancer progression remains unclear.

Recent studies have demonstrated that REST-003, a noncoding RNA, is expressed at low levels in weakly invasive breast cancer cells but at elevated levels in highly invasive cancer cells, and REST-003 expression is controlled by an uncharacterized serine/arginine repeat-related protein (SRRM3) (13). The research revealed that REST-003 and SRRM3 played an important role in regulating cell invasiveness and emphasized their potential novel therapeutic value in breast cancer.

In the present study, we hypothesized that RYBP may function as a novel molecular target in breast cancer therapy. We investigated the expression level of RYBP and explored the effect of RYBP overexpression on tumor growth and metastasis in both breast cancer cell lines and in a nude mouse model. We also analyzed the potential molecular mechanism involved in this process.

\section{Materials and methods}

Cell culture. Normal 76N and tumor SK-BR-3, ZR-75-1, T47D, MDA-MB-231 and MCF-7 breast cells were used in this study. All cells were purchased from the Type Culture Collection of the Chinese Academy of Sciences (Shanghai, China). Cells were maintained in Dulbecco's modified Eagle's medium (DMEM) containing 10\% fetal bovine serum (10\% DMEM) and cultured in $5 \% \mathrm{CO}_{2}$ at $37^{\circ} \mathrm{C}$.

Patients and samples. Thirty-three paired primary tumors and matched peritumoral tissues of breast cancer samples were collected from patients who underwent surgical resection in the First Affiliated Hospital of Sun Yat-sen University, Guangzhou, China, between January 2014 and January 2016. Patients diagnosed with distant metastasis were excluded.

Quantitative RT-PCR ( $q R T-P C R)$. Total RNA was extracted from pretreated cells using TRIzol reagent (Life Technologies, Carlsbad, CA, USA) according to the manufacturer's protocol. The RNA samples were reverse-transcribed into cDNA using the PrimeScript ${ }^{\mathrm{TM}}$ RT reagent kit (Takara Co., Ltd., Tokyo, Japan). The resulting cDNAs were amplified with GoTaq qPCR Master Mix (Promega, Madison, WI, USA) following standard procedures, and the experiment was performed on a MiniOpticon $^{\mathrm{TM}}$ real-time PCR detection instrument (Bio-Rad Laboratories, Hercules, CA, USA). The primers used in this study are shown in Table I.

Immunohistochemistry (IHC). Breast cancer tissues and the paired peritumoral tissues were fixed in $10 \%$ formalin and embedded in paraffin. The blocks were cut into 4-mm sections and then dewaxed and hydrated. After endogenous peroxidase was blocked with $3 \%$ hydrogen peroxide, the sections were incubated with RYBP primary antibody (HPA053357; Sigma-Aldrich, Schnelldorf, Germany) and diluted 1:500 at $4^{\circ} \mathrm{C}$ overnight. Subsequently, the sections were washed to remove unbound antibody and incubated with the secondary
Table I. Primers used in the present study.

\begin{tabular}{ll}
\hline Primer name & \multicolumn{1}{c}{ Sequence (5'-3') } \\
\hline H-REST F & GAACTCATACAGGAGAACGCC \\
H-REST R & GAACTGCCGTGGGTTCACA \\
H-REST003 F & AGTGTCGGGGCGACTCCCG \\
H-REST003 R & GGCATTCCTAACTGAAATAGG \\
H-SRRM3 F & TCCTGGAGCTCCAGCCGCTCGCCC \\
H-SRRM3 R & CTCAGAGTGCCTTGCGCGGCCCTCG \\
H-RYBP-F & CGCAGACGAAGGGTTTTGG \\
HRYBP-R & GAATTGATCCGAGGTTTTCTGGT \\
H-GAPDH-F & GAGTCAACGGATTTGGTCGT \\
H-GAPDH-R & GACAAGCTTCCCGTTCTCAG
\end{tabular}

antibody (sc-2055, 1:10,000; Santa Cruz Biotechnology, Santa Cruz, CA, USA) at room temperature for $1 \mathrm{~h}$. The sections were visualized using the Vectastain ABC Peroxidase Elite kit (Vector Laboratories, Burlingame, CA, USA) and 3,3'-diaminobenzidine (DAB) tetrahydrochloride (Sigma-Aldrich) as a substrate.

Western blot analysis. Fresh breast cancer tissues and paired peritumoral tissues were washed with phosphate-buffered saline (PBS) and stored at $-80^{\circ} \mathrm{C}$ after surgical resection. Cell lysates were prepared using the NucBuster ${ }^{\mathrm{TM}}$ Protein Extraction kit (Novagen, Darmstadt, Germany) according to the manufacturer's instructions. The protein concentration was quantified using the Bradford assay, separated by $10 \%$ SDS-PAGE electrophoresis and transferred to PVDF membranes. The PVDF membranes were blocked with 5\% skim milk powder for $1 \mathrm{~h}$ at room temperature and then incubated with primary antibody against RYBP (1:400 dilution), E-cadherin (sc-7870, 1:2,000 dilution; Santa Cruz Biotechnology), snail (sc-393172, 1:200 dilution; Santa Cruz Biotechnology), cyclin A (ab38, 1:1,000 dilution; Abcam, Cambridge, MA, USA) and cyclin B1 (ab32053, 1:2,500 dilution; Abcam) at $4^{\circ} \mathrm{C}$ overnight. The samples were then incubated with the secondary antibody (sc-2006, 1:1,000 dilution; Santa Cruz Biotechnology) at room temperature for $1 \mathrm{~h}$, and the blots were visualized using chemiluminescence (ECL; Forevergen Biosciences Center, Guangzhou, China). GAPDH (sc-365062, 1:1,000 dilution; Santa Cruz Biotechnology) was used as a reference.

Stable cell line generation and transfection. RYBP (Gene ID: 23429) was cloned into the EcoRI and XbaI sites of the LV-003 lentivirus vector (Forevergen Biosciences Center). The resulting lentivirus vector was co-transfected with packaging vectors into HEK-293T cells, and the cell supernatant was then collected after $48 \mathrm{~h}$ of transfection and subjected to ultracentrifugation at $25,000 \mathrm{rpm}$ for $1.5 \mathrm{~h}$. The lentivirus was then re-suspended in PBS, and 5-10 $\mu \mathrm{g} / \mathrm{ml}$ of polybrene was added to the RYBP lentivirus for MDA-MB-231 and SK-BR-3 cell infection. After a 48-h incubation, the cells were cultured in $10 \%$ DMEM with $2 \mu \mathrm{g} / \mathrm{ml}$ puromycin for $72 \mathrm{~h}$ to generate MDA-MB-231-RYBP and SK-BR-3-RYBP cells. The cell cycle distribution, cell migration and cell invasion 
of MDA-MB-231-RYBP and SK-BR-3-RYBP cells were then analyzed to evaluate cell growth and metastasis.

RYBP (Gene ID: 23429) and SRRM3 (Gene ID: 222183) was cloned into the $E c o$ RI and $X b a \mathrm{I}$ sites of the pCMV vector. The si-SMMR3 (5'-AGAAGAAGAGUGUGAAGAAUU-3') and si-REST003 (5'-GCAGCAGCACGGAGGAAUU-3') were purchased from Shanghai GenePharma, Co., Ltd., (Shanghai, China). Cells were seeded into 6 -well plates $12 \mathrm{~h}$ prior to transfection. A final concentration of $100 \mathrm{nM}$ of siRNA (Shanghai GenePharma) and $2 \mu \mathrm{g}$ of plasmid were transfected into the cells according to standard procedures. After $48 \mathrm{~h}$ of culture, the cells were collected for mRNA expression and cell metastasis analysis.

Clonogenic assay of cells in vitro. After treatment, the cells were seeded into 6-well plates at a density of $1.5 \times 10^{5}$ cells/well and cultured in 10\% DMEM. The cell colonies were fixed with glutaraldehyde $(6.0 \% \mathrm{v} / \mathrm{v})$, stained with crystal violet $(0.5 \% \mathrm{w} / \mathrm{v})$ and counted after 15 days. Clone formation ratios were calculated as colonies/total cells. A colony was defined as consisting of at least 50 cells (14). The experiments were repeated three times.

MTS assay. Cell viability was measured using the MTS [3-(4,5-dimethylthiazol-2-yl)-5-(3-carboxymethoxyphenyl)2-(4-sulfophenyl)-2H-tetrazolium] assay. The cells were cultured in 96-well flat-bottomed plates at a density of $1 \times 10^{3}$ cells/well $24 \mathrm{~h}$ prior to the MTS assay. The cells were then stained with MTS reagent in 10\% DMEM for $4 \mathrm{~h}$ and subjected to a microplate reader (Diatek) to determine the OD value of each well at a wavelength of $490 \mathrm{~nm}$. Three biological replicates were evaluated.

Flow cytometry. Stable RYBP-overexpressing cell lines (MDA-MB-231-RYBP and SK-BR-3-RYBP) and their corresponding cells (MDA-MB-231-NC and SK-BR-3-NC) were fixed with $1 \mathrm{ml}$ of $70 \%$ ice-cold ethanol overnight at $4^{\circ} \mathrm{C}$. The cells were washed twice with PBS and centrifuged for $10 \mathrm{~min}$ at $1,000 \mathrm{rpm}$; the cell pellets were then stained with $50 \mu \mathrm{g} / \mathrm{ml}$ of propidium iodide and $100 \mathrm{U} / \mathrm{ml}$ of RNase A, and incubated in PBS for $30 \mathrm{~min}$ at room temperature in the dark. A flow cytometer (BD Biosciences, San Jose, CA, USA) with CellQuest software was used to analyze the cell cycle distribution, and the proportions of cells in different phases of the cell cycle were determined based on the DNA content.

Transwell assay. Cell migration and invasion assays were performed using the Transwell assay (Transwell; Cambridge, MA, USA) (15). The cells were cultured in the upper compartment of the Transwell at a density of $2 \times 10^{4}$ cells $/ 50 \mu \mathrm{l} / \mathrm{well}$. The cells in the upper compartment migrated to the lower compartment through an $8 \mu \mathrm{m}$ membrane (for the migration assay) or a Matrigel-coated membrane (for the invasion assay) (Becton-Dickinson, Bedford, MA, USA). Cells in the upper compartment were cultured in serum-free DMEM, while $10 \%$ DMEM was added to the lower compartment. After $48 \mathrm{~h}$ of cell culture, the cells in the upper compartment were removed, and the migrated/invasive cells in the lower compartment were fixed in methanol and stained with $10 \%$ Giemsa solution (Sigma-Aldrich). The cell numbers in four high-power fields were counted under a microscope. Three biological replicates were evaluated.

Nude mouse model. For tumor xenograft model establishment, 4- to 6-weeks-old BALB/c nude mice were obtained from the Animal Center Laboratory of Sun-Yat sen University. The Institute Research Medical Ethics Committee of Sun Yat-sen University granted approval for this study. Approximately 5x10 ${ }^{6}$ MDA-MB-231-RYBP, SK-BR-3-RYBP, and their control cells (MDA-MB-231-NC and SK-BR-3-NC) were subcutaneously injected into each group ( $\mathrm{n}=5$ /group) (16). The tumor volume and general physical condition of the mice were monitored every 6 days. The tumor volume was calculated as the width ${ }^{2} \mathrm{x}$ length/2. After 42 days of modification, the mice were sacrificed and the xenograft tumors were removed. For the nude mouse model of breast cancer metastasis to the lung, 5x10 ${ }^{6}$ MDA-MB-231-RYBP, SK-BR-3-RYBP and their control cells (MDA-MB-231-NC and SK-BR-3-NC) were injected into the mammary fat pad of mice as previously described (17) $(n=5$ per group). Six weeks after the cell implantation, when signs of disease (weight loss and decreased grooming) were observed in the mice, the lungs of each mouse were dissected, and metastatic foci in the lungs were counted. Hematoxylin and eosin (H\&E) staining was also performed to facilitate review.

Ethical standards. All human studies were approved by Sun Yat-sen University. All human studies were performed in accordance with the 1975 Helsinki Declaration and its subsequent amendments. All subjects signed an informed consent form prior to inclusion in the study.

Statistical analysis. The SPSS 18.0 software (IBM, Armonk, NY, USA) was used for the statistical analysis. The results are presented as the mean $\pm \mathrm{SD}$. The Student's t-test method was used to analyze differences between the two groups. For the disease-free survival (DSF) analysis, the expression data for RYBP mRNA z-Scores (RNA Seq V2 RSEM) from 350 cases were downloaded from The Cancer Genome Atlas (TCGA) dataset [Breast Invasive Carcinoma (TCGA, Cell 2015)] and analyzed using GraphPad Prism v.6 (Graphpad Software Inc., La Jolla, CA, USA). DSF curves were generated using the Kaplan-Meier method, and the log-rank Chi-square test was used to analyze the significance of the correlation between patients with high and low RYBP expression. $\mathrm{P}<0.05$ was considered a statistically significant difference.

\section{Results}

RYBP is downregulated in clinical samples of breast cancer patients and cell lines and is associated with poor survival in breast cancer patients. To determine whether the expression of RYBP was altered in breast cancer tumors, qPCR and western blot analysis were conducted. As shown in Fig. 1, both RYBP mRNA (Fig. 1A) and protein (Fig. 1B) were downregulated in the tumor tissues compared with the peritumoral tissues of breast cancer patients.

To assess whether RYBP expression is associated with longer survival in breast cancer patients, DFS was determined in the TCGA dataset breast cancer patient cohort. The survival analysis showed that DFS differed between patients with high 
A
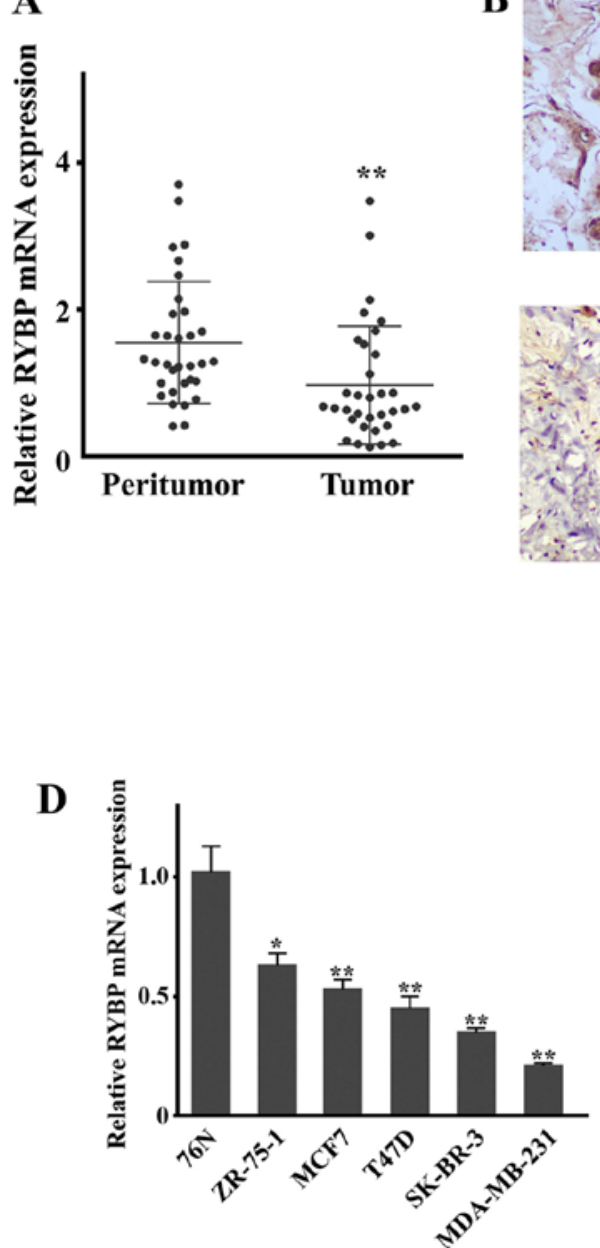

B
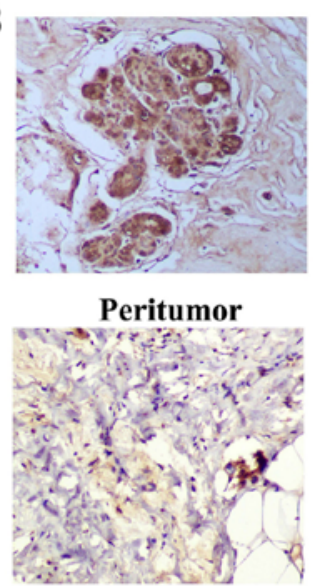

Tumor
C

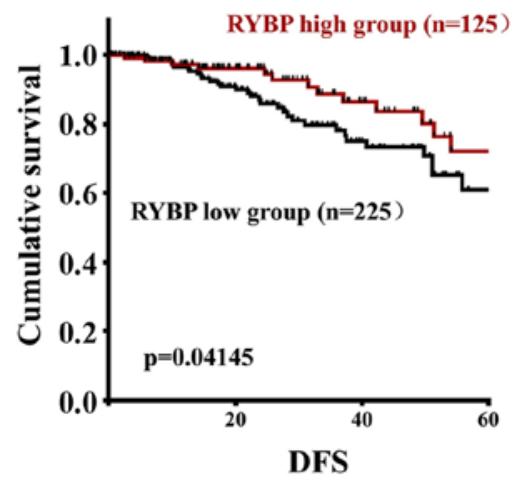

$\mathbf{E}$
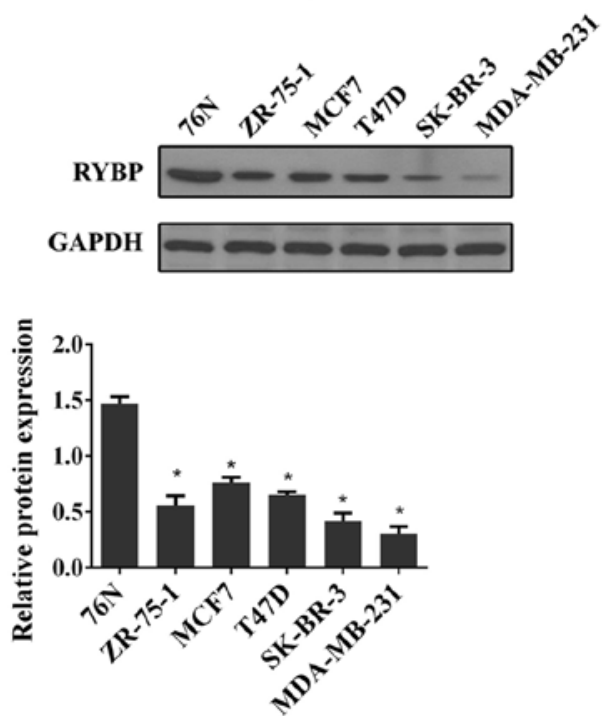

Figure 1. Expression of RYBP in breast cancer clinical samples and cell lines and its association with DFS durations. (A) qRT-PCR results of the relative expression of RYBP mRNA in peritumor and tumor tissues of breast cancer patients $(\mathrm{n}=33)$. ${ }^{* *} \mathrm{P}<0.01$ vs. peritumor. (B) Representative sample of peritumor and tumor tissues detected by IHC. (C) Kaplan-Meier plots of DFS in the TCGA cohort according to the expression of RYBP. (D) Relative expression of RYBP mRNA (E) and protein in normal 76N and SK-BR-3, ZR-75-1, T47D, MDA-MB-231 and MCF-7 tumor cells. Results represent 3 independent experiments. ${ }^{*} \mathrm{P}<0.05$ vs. $76 \mathrm{~N},{ }^{* *} \mathrm{P}<0.01$ vs. $76 \mathrm{~N}$.

and low RYBP expression. Patients with high RYBP expression had significantly longer DFS than those with low RYBP expression (Fig. 1C).

The expression levels of RYBP mRNA (Fig. 1D) and protein (Fig. 1E) were also evaluated in normal $76 \mathrm{~N}$ and tumor SK-BR-3, ZR-75-1, T47D, MDA-MB-231 and MCF-7 breast cells. As shown in Fig. 1D and E, the qPCR and western blot results showed that RYBP was downregulated in all analyzed breast tumor cells. Of these cells, SK-BR-3 and MDA-MB-231 cells exhibited the first and second lowest expression, and were therefore selected for subsequent analysis of RYBP function.

Overexpression of RYBP suppresses cell growth and metastasis in $M D A-M B-231$ and $S K-B R-3$. The differential expression of RYBP between tumorous and peritumor breast cancer tissues led us to investigate its role in breast cancer development and metastasis. Because RYBP was significantly downregulated in SK-BR-3 and MDA-MB-231 cells, we applied lentiviral-mediated overexpression of RYBP in these two tumor cell lines. As shown in Fig. 2A, lentivirus-mediated stable RYBP overexpressing cell lines, MDA-MB-231-RYBP and SK-BR-3-RYBP, were established, as evidenced by the upregulation of RYBP and apparent in the western blots compared with their corresponding negative controls of MDA-MB-231-NC and SK-BR-3-NC cells. Cell proliferation was then determined using clonogenic and MTS assays. The clonogenic assay of cells showed that RYBP-overexpressing cells (MDA-MB-231-RYBP and SK-BR-3-RYBP) had a lower rate of clone formation compared with $\mathrm{NC}$ cells (Fig. 2B). The MTS assay also revealed consistently decreasing OD values in MDA-MB-231-RYBP and SK-BR-3-RYBP cells compared with negative control cells (Fig. 2C). Furthermore, flow cytometric analysis of the cell cycle distribution revealed a higher proportion of S-phase cells in MDA-MB-231-RYBP compared with MDA-MB-231-NC cells. A higher proportion of S-phase cells was also observed in SK-BR-3-RYBP cells (Fig. 2D).

Transwell assays were performed to evaluate the effects of RYBP overexpression on cell migration and invasive ability in breast cancer cells. As shown in Fig. 3A and B, the number of migrated cells and invasive cells in MDA-MB-231-RYBP was significantly lower than that in MDA-MB-231-NC. Similar results were observed in SK-BR-3-RYBP cells.

Western blot analysis of proliferation-related proteins (cyclin A and cyclin B1) and EMT-related markers 

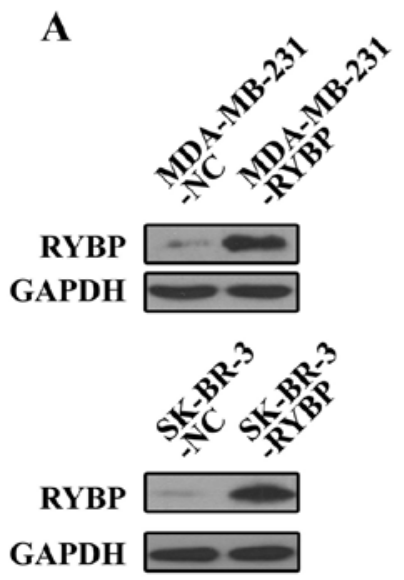

D
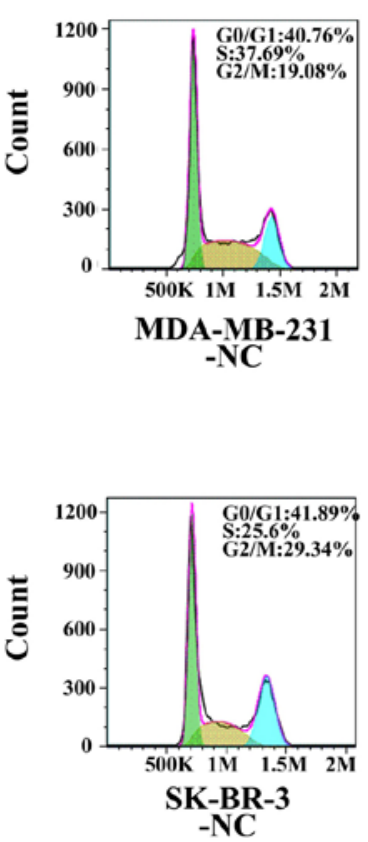

B
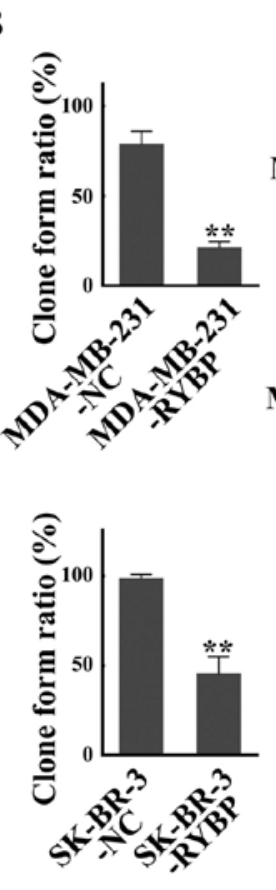
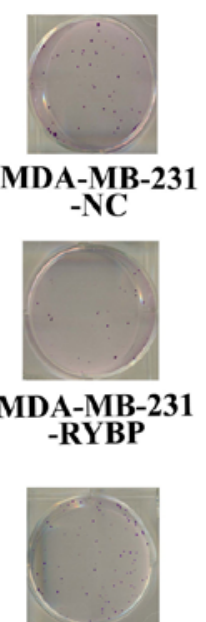

SK-BR-3

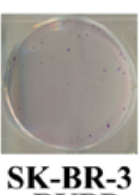

$\underset{\text {-RYBP }}{\text { SK-BR-3 }}$

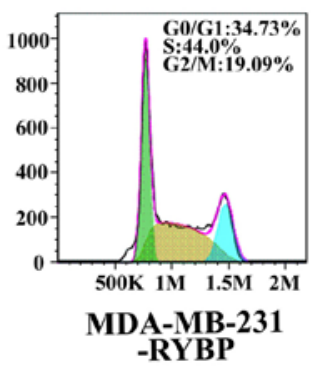

-RYBP

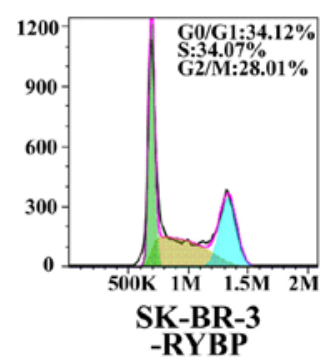

C
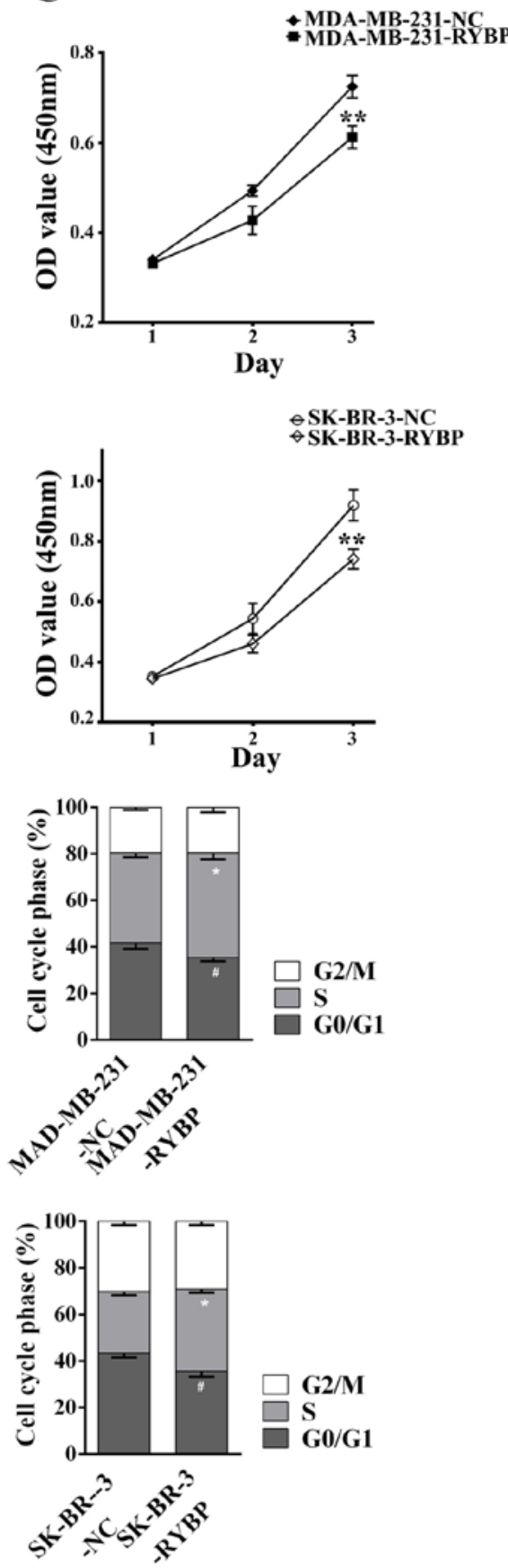

Figure 2. Overexpression of RYBP suppresses cell growth in MDA-MB-231 and SK-BR-3 cells. (A) Western blot analyis demonstrating overexpression of RYBP in RYBP-overexpressing MDA-MB-231 (MDA-MB-231-RYBP) and SK-BR-3 (SK-BR-3-RYBP) cells. MDA-MB-231-NC and SK-BR-3-NC were the corresponding negative control cells. (B) Clonogenic assay of MDA-MB-231-RYBP and SK-BR-3-RYBP cells. The results are represented as the clone form ration. (C) The OD values for MDA-MB-231-RYBP and SK-BR-3-RYBP cells were determined using the MTS assay. (D) Cell cycle distribution in each group; cell proportions in each cell cycle phase were evaluated. ${ }^{* *} \mathrm{P}<0.01$ vs. MDA-MB-231-NC or SK-BR-3-NC. ${ }^{*} \mathrm{P}<0.05$ vs. S-phase of the NC group, ${ }^{\prime \prime} \mathrm{P}<0.05$ vs. GO/G1-phase of the NC group.

(E-cadherin and snail) revealed that E-cadherin was upregulated while snail, cyclin A and cyclin B1 were downregulated in MDA-MB-231-RYBP and SK-BR-3-RYBP cells (Fig. 3C and D).

Overexpression of RYBP suppresses cell growth and metastasis of MDA-MB-231 and SK-BR-3 cells in nude mice. Cell growth and metastasis were further analyzed in nude mice. MDA-MB-231-RYBP and MDA-MB-231-NC cells were xenografted subcutaneously into nude mice. Twelve days after subcutaneous implantation, the nude mice bearing MDA-MB-231-RYBP tumors showed a significantly lower tumor volume compared with the mice with MDA-MB-231-NC cell implantation. A smaller tumor size and lower tumor weight were also observed in the nude mice with subcutaneous MDA-MB-231-RYBP implantation after 42 days (Fig. 4A).

To assess the effect of RYBP on tumor metastasis in vivo, MDA-MB-231-RYBP cells and negative control cells 

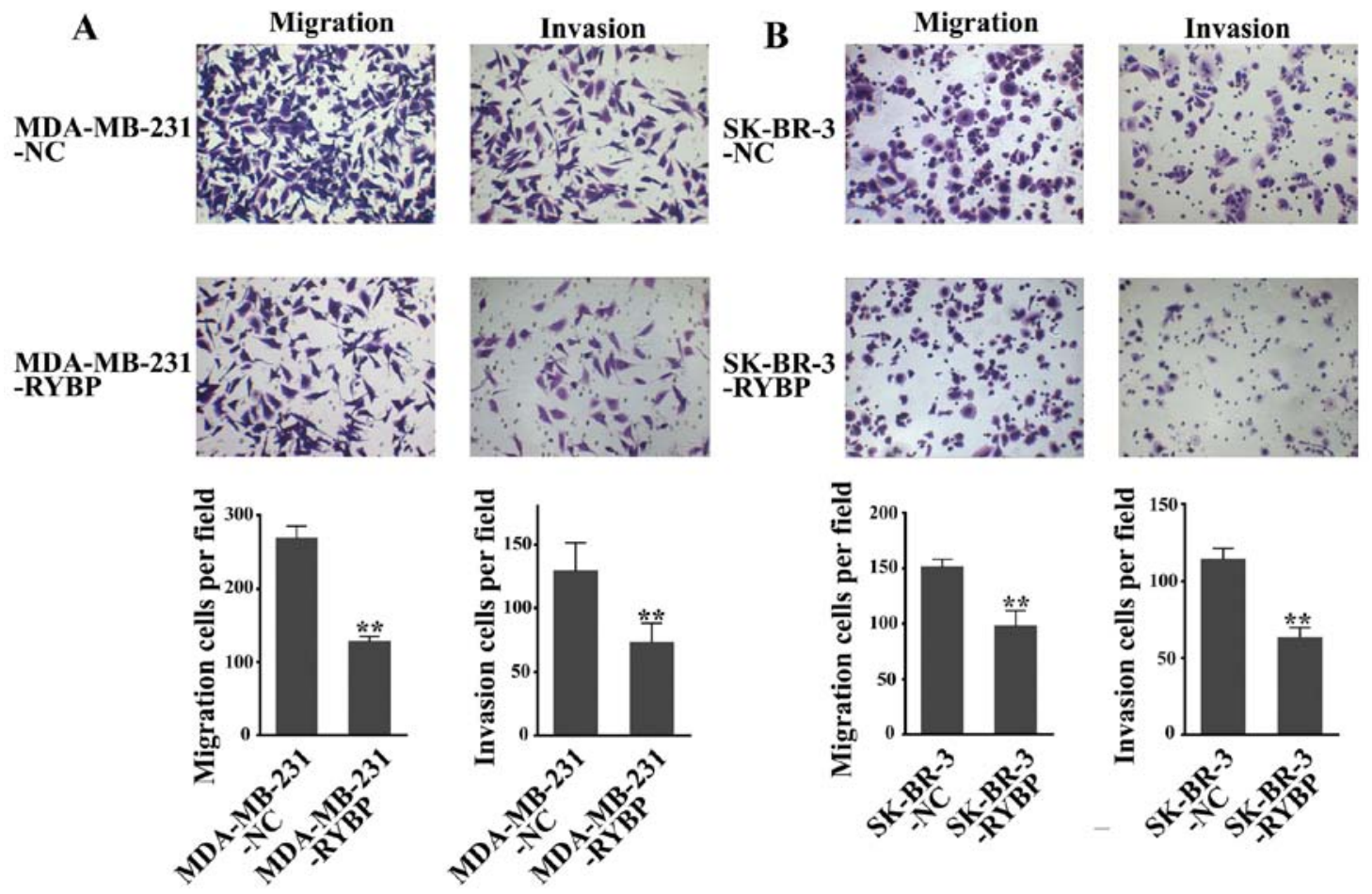

C

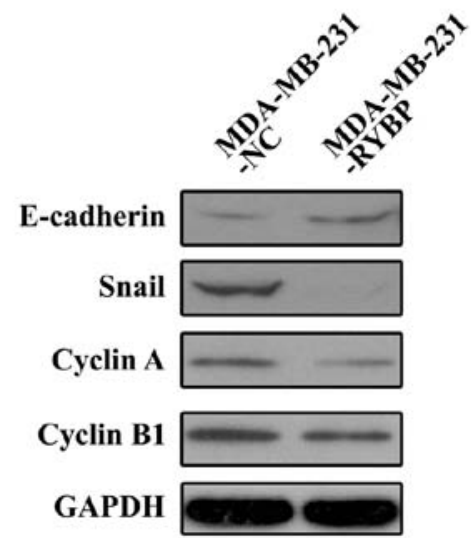

D

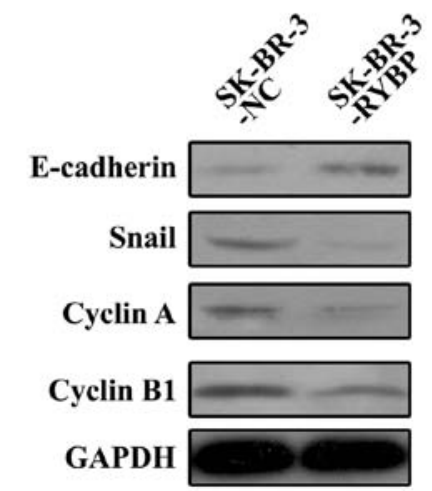

Figure 3. Overexpression of RYBP suppresses cell migration and invasion in MDA-MB-231 and SK-BR-3 cells. (A) The numbers of migrating and invasive cells was detected and compared between MDA-MB-231-RYBP and MDA-MB-231-NC cells. (B) Migrating and invasive cells were detected and compared between SK-BR-3-RYBP and SK-BR-3-NC cells. Cell proliferation-related proteins (cyclin A and cyclin B1) and EMT-related markers (E-cadherin and snail) were analyzed by western blotting in MDA-MB-231-RYBP (C) and SK-BR-3-RYBP (D) cells. ${ }^{* *}$ P<0.01 vs. MDA-MB-231-NC or SK-BR-3-NC.

(MDA-MB-231-NC) were injected into the mammary fat pads of mice to mimic the metastatic process in breast cancer patients. Six weeks after cell implantation, the cells metastasized to the lungs. Metastatic foci were observed in the lungs of MDA-MB-231-NC-treated nude mice. However, barely any metastatic foci were observed in the lungs of MDA-MB-231RYBP-treated nude mice. H\&E staining revealed a mass of tumor cells in the lungs of MDA-MB-231-NC mice, whereas reduced metastases were observed in the lungs of MDA-MB-231-RYBP-treated nude mice (Fig. 4B).

Knockdown of SRRM3, which is downregulated after RYBP overexpression, suppresses cell growth and metastasis in MDA-MB-231 and SK-BR-3 cells. SRRM3 has been reported to suppress MDA-MB-231 Matrigel invasiveness (13), and therefore its role was investigated in the present study. As shown in Fig. 5A, the qPCR results showed that SRRM3 was downregulated in both RYBP-overexpressing cell lines (MDA-MB-231-RYBP and SK-BR-3-RYBP). The Transwell assay showed that knockdown of $S R R M 3$ mRNA expression by siRNA in MDA-MB-231 and SK-BR-3 cells resulted in a significant reduction of the quantity of migrated and invasive (Fig. 5B and C). Cell growth inhibition was observed using the MTS assay. The OD values were significantly lower in the si-SRRM3-treated MDA-MB-231 and SK-BR-3 cells compared with the si-NC-treated cells (Fig. 5D).

REST-003 ncRNA levels are downregulated in RYBPoverexpressing and SRRM3-inhibited cells. REST-003 ncRNA is thought to be regulated by SRRM3 (13). Determination of REST-003 ncRNA expression by qRT-PCR revealed that REST-003 ncRNA was downregulated in MDA-MB-231- 


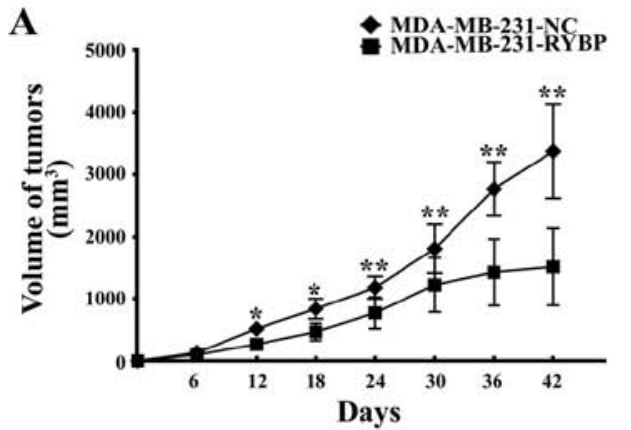

B

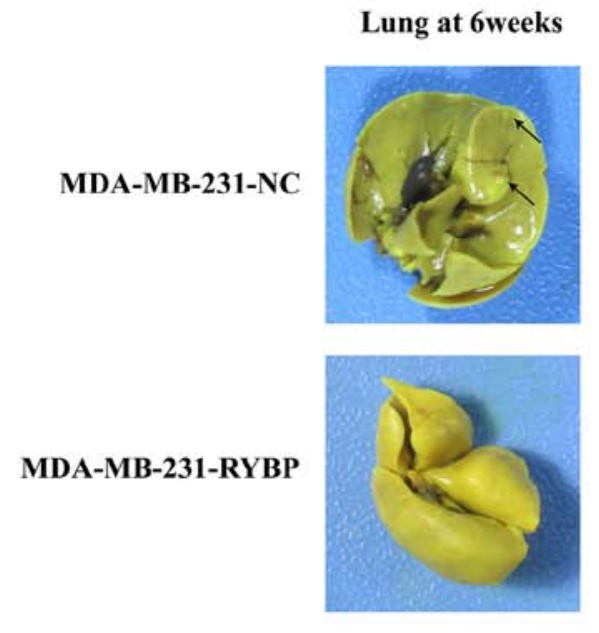

MDA-MB-231-NC

MDA-MB-231-RYBP

HE 40X
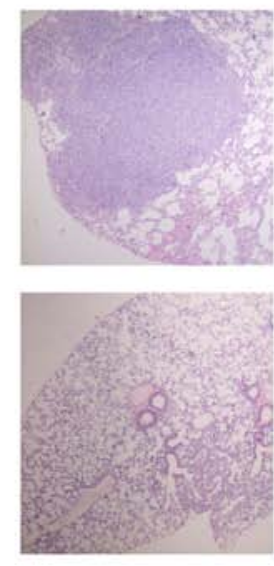
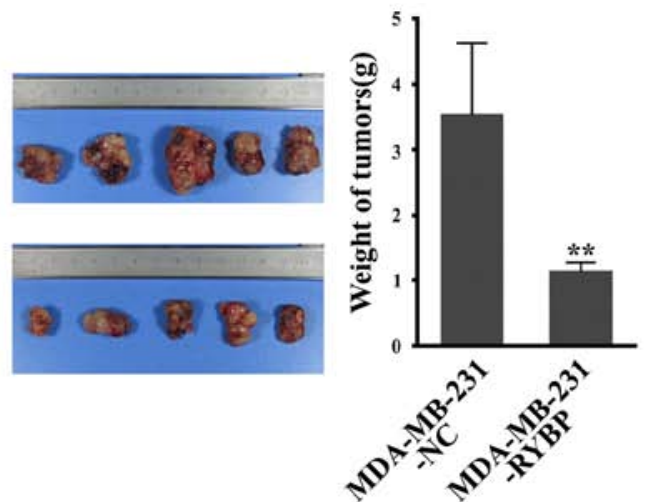

HE 200X
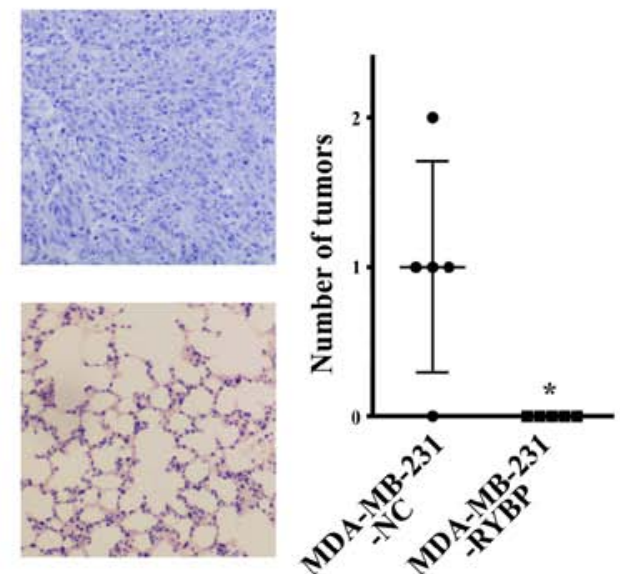

Figure 4. Overexpression of RYBP suppresses cell growth and metastasis in nude mice. (A) The tumor volume of MDA-MB-231-RYBP and MDA-MB-231-NC cell xenografted nude mice was measured every 6 days. Xenografted tumors were resected, and the tumor weight in each group was calculated after 6 weeks of implantation. ${ }^{* *} \mathrm{P}<0.01$ vs. MDA-MB-231-NC. (B) Representative lung metastasis image and H\&E staining image of MDA-MB-231-RYBP and MDA-MB-231-NC cells in xenografted nude mice at week 6. Metastatic foci in each group were calculated.

RYBP and SK-BR-3-RYBP cells (Fig. 6A). Because SRRM3 downregulation was also observed after RYBP overexpression, as mentioned above, the expression of REST-003 ncRNA was subjected to further analysis in si-SRRM3 cells. As shown in Fig. 6B, REST-003 ncRNA was significantly reduced in the si-SRRM3 cells of MDA-MB-231 and SK-BR-3 cells when compared with the negative control si-NC cells.

RYBP overexpression suppresses cell migration, invasion and growth by downregulating SRRM3/REST-003. To further explore the molecular mechanisms of RYBP, SRRM3 and REST-003 in breast cancer, we constructed an RYBP and SRRM3-overexpressing plasmid (PCMV-RYBP and PCMV-SRRM3). We also downregulated REST003 expression in the cells that were transfected with PCMV-RYBP and PCMV-SRRM3. The results revealed that overexpression of RYBP suppressed the migration and invasion of tumor cells (MDA-MB-231 and SK-BR-3), and increased tumor cell migration and invasion was observed in the PCMV-RYBP+PCMV-SRRM3+siNC group compared with the PCMV-RYBP+siNC group (Fig. 7A). Furthermore, cell migration and invasion were suppressed in the pCMVRYBP+pCMV-SRRM3+siREST003 group compared with the pCMV-RYBP+pCMV-SRRM3+siNC group (Fig. 7A). The MTT assay showed similar results. A higher OD value was obtained for MDA-MB-231 and SK-BR-3 cells in the
PCMV-RYBP+PCMV-SRRM3+siNC group compared with the pCMV-RYBP+siNC group, and a lower value was determined for the pCMV-RYBP+pCMV-SRRM3+siREST003 group (Fig. 7B). These results demonstrated that RYBP could influence the migration and growth of breast cancer cells by downregulating SRRM3/REST003. This molecular mechanism plays an important role in the function of breast cancer cells.

\section{Discussion}

RYBP belongs to the PcG family of proteins and is considered to function as a transcriptional suppressor via epigenetic modification of chromatin (18). Downregulated expression of RYBP has been reported in HCC cell lines and clinical samples. In the present study, we enhanced our knowledge of the expression of RYBP in breast cancer and demonstrated that RYBP is downregulated in breast cancer cell lines (SK-BR-3, ZR-75-1, T47D, MDA-MB-231 and MCF-7), as well as in clinical breast cancer samples.

To date, the inhibitory role of RYBP in tumor progression has only been confirmed in HCC and NSCLC. Low expression levels of RYBP have been shown to correlate with a poor prognosis in patients with HCC and NSCLC and are also associated with shorter survival durations in patients with cervical cancer who received chemoradiotherapy $(8,11,12)$. By overexpressing 
A

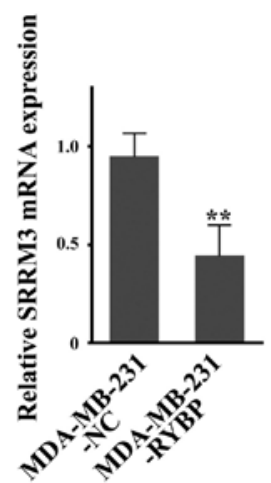

C

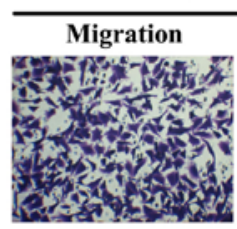

MDA-MB-231

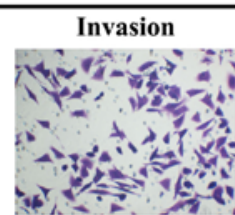

Si-SRRM3
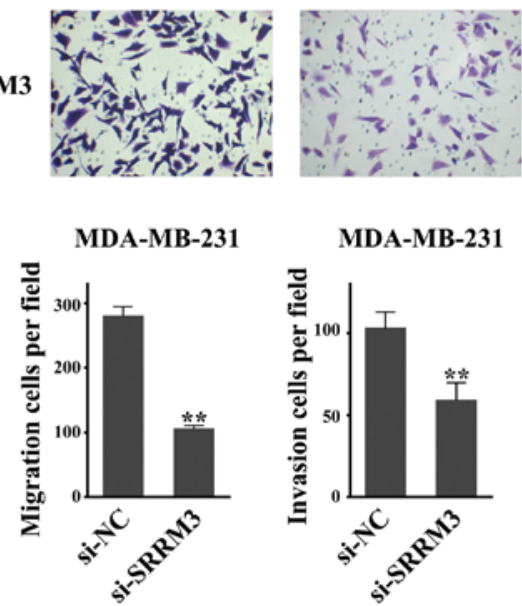

D

MDA-MB-231

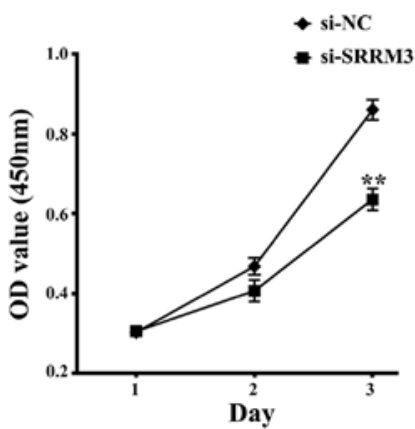

B
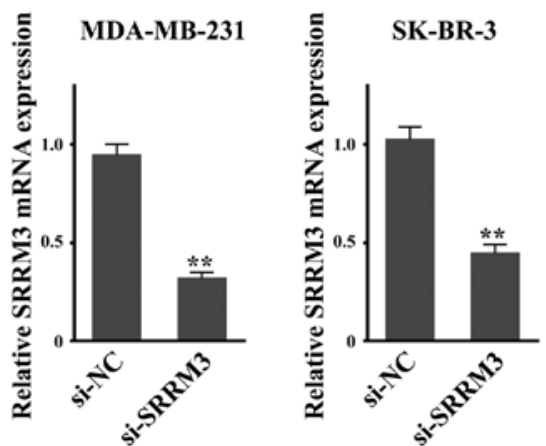

SK-BR-3
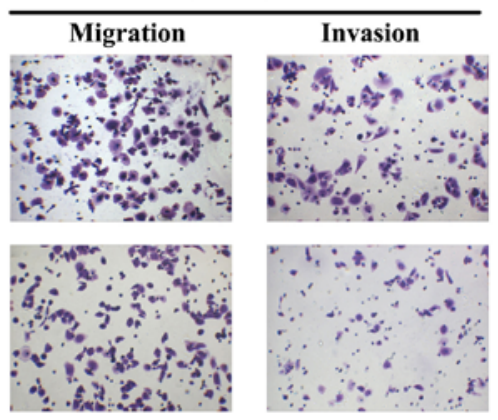

SK-BR-3
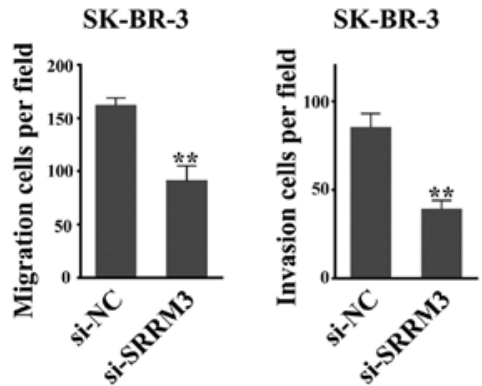

SK-BR-3

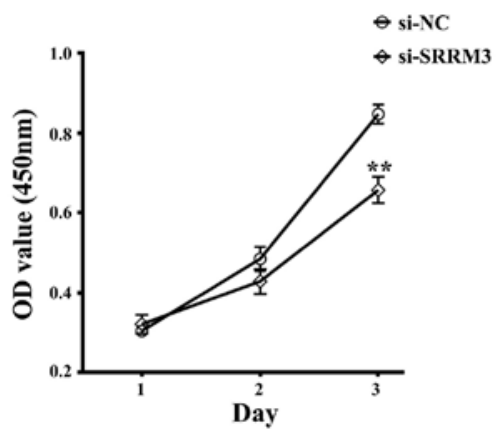

Figure 5. SRRM3 is involved in RYBP-mediated cell growth and metastasis inhibition in MDA-MB-231 and SK-BR-3 cells. (A) qRT-PCR detection of the relative expression levels of SRRM3 in MDA-MB-231-RYBP and SK-BR-3-RYBP cells. (B) Knockdown of SRRM3 mRNA using siRNA reduced SRRM3 expression in MDA-MB-231 and SK-BR-3 cells. (C) Cell migration and invasion in SRRM3 knockdown cells (si-SRRM3) and negative control cells (si-NC) were assessed using the Transwell assay. The migrated and invasive cells per field were counted. (D) The OD values in si-SRRM3 and si-NC cells were determined using the MTS assay.

RYBP, we observed suppressed cell proliferation, migration and invasion in two breast cancer cell lines (MDA-MB-231 and SK-BR3). Our results for the nude mouse xenograft tumor experiment as well as our assessment of metastatic tumor foci, showed that RYBP suppressed MDA-MB-231 cell growth and metastasis in vivo. Survival analysis of breast cancer patients with low or high RYBP expression provided additional support for the contention that high RYBP expression correlates with longer 5-year survival rates in breast cancer patients. Taken together, these animal model and survival studies suggest that increased RYBP expression plays a demonstrable role in the repression of tumor growth and metastasis in breast cancer.

Analysis of the cell cycle distribution by flow cytometry revealed that cells were arrested at the S phase in the RYBP- 

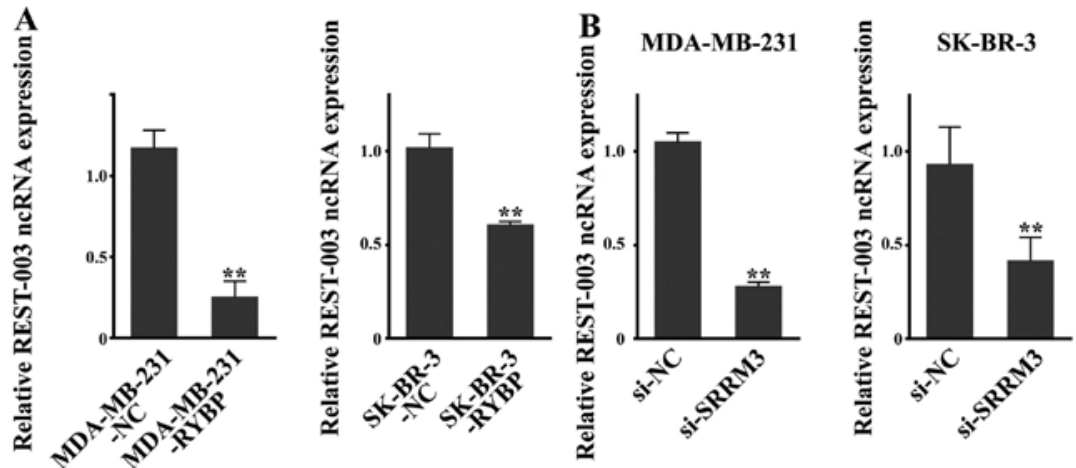

Figure 6. qRT-PCR analysis of RES-003 in RYBP-overexpressing cells (A) and SRRM3-suppressed cells (B).
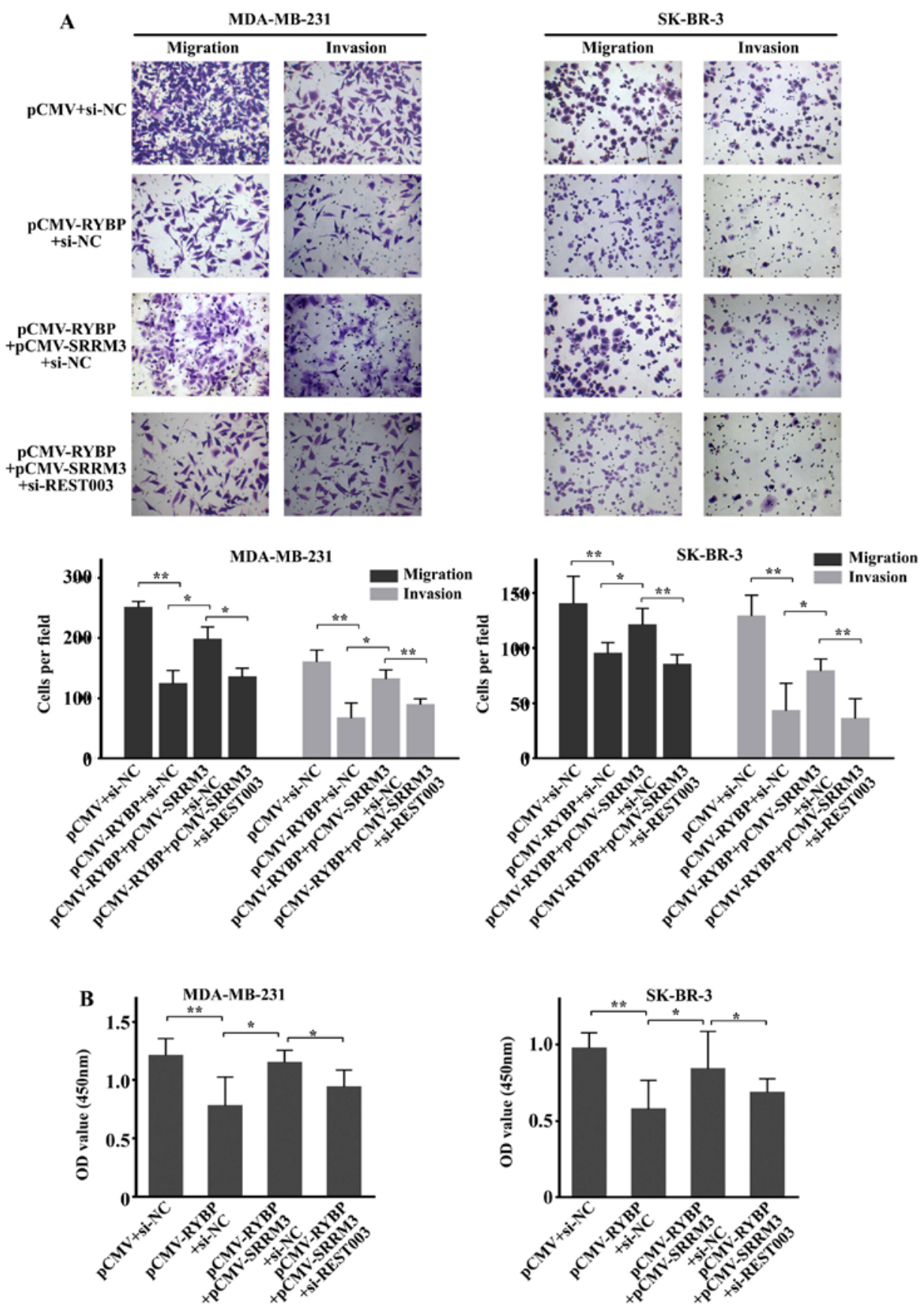

Figure 7. RYBP overexpression suppresses cell migration, invasion and growth by downregulating SRRM3/REST-003. Cell migration, invasion (A) and growth (B) was reduced in the pCMV-RYBP+pCMV-SRRM3+siNC group compared with the pCMV-RYBP+siNC group and increased in the pCMV-RYBP+ pCMV-SRRM3+siREST003 group. 
overexpressing groups, which suggests that $\mathrm{S}$-phase arrest contributes to the observed cell growth inhibition by RYBP. Cyclin A levels normally peak in G2/M-phase of the cell cycle. Cyclin B1 has been reported to be involved in regulating the initiation of mitosis, and increased expression of cyclin B1 has been suggested to be a negative prognostic marker of proliferation in breast cancer (19-21). Downregulation of cyclin A and cyclin B1 expression was confirmed by western blot analysis, and these results suggested that RYBP disrupted the cell cycle and inhibited proliferation.

Epithelial-mesenchymal transition (EMT) has been found to regulate breast cancer cell metastasis. EMT converts epithelial cells into migratory cells that are able to traverse the extracellular matrix. This process results in a loss of epithelial cell traits and a gain of the properties of mesenchymal cells (22), leading to the establishment of distant metastases $(23,24)$. E-cadherin and Snail are important markers of the EMT process. Research has showed that a central event in EMT is the downregulation of E-cadherin expression, which leads to a loss of cell-to-cell contact (25). Additionally, the transcription factor Snail has been reported to be a major suppressor of E-cadherin in many tumor types. Increased Snail expression leads to more aggressive tumor features, which enhances tumor cell migration and invasion $(26,27)$. Our evaluation of E-cadherin and snaill revealed upregulated E-cadherin expression and reduced activation of snaill in RYBP-overexpressing breast cancer cells, suggesting a potential role for RYBP in reducing invasive and migrating phenotypes in breast cancer cells. Thus, E-cadherin and snaill may be involved in reversing the EMT process.

The mRNA abundance of SRRM3 was decreased in response to RYBP overexpression. Reduced cell growth and migration as well as invasive ability were also observed in RYBP-overexpressing MDA-MB-231 and SK-BR-3 cells (13), and our results confirmed the ability of SRRM3 to promote cell invasion and cell migration in breast cancer. Moreover, because SRRM3 was downregulated in RYBP-overexpressing cells, we constructed an SRRM3-overexpressing plasmid (pCMVSRRM3) and transfected it into the RYBP-overexpressing cells lines. The results revealed increased migration and growth of cancer cells, suggesting that RYBP may regulate SRRM3 and thus inhibit breast cancer cell metastasis.

An alternatively spliced RE1-silencing transcription (REST) factor, REST-003, which is regulated by SRRM3, has been shown to be positively associated with breast cancer cell invasion (13). We found a reduced mRNA abundance of REST-003 in response to RYBP overexpression. Downregulation of SRRM3 resulted in a decrease in REST-003 ncRNA, consistent with the results of a previous study (13). We also observed suppressed migration and growth in response to REST-003 downregulation in cells transfected with pCMV-RYBP and pCMV-SRRM3 compared with the pCMV-RYBP+pCMV-SRRM+siREST003 group. Thus, cell migration and growth were promoted following the upregulation of REST-003 by SRRM3 overexpression, and this process could be suppressed by the downregulation of REST-003. These results suggest that REST-003 ncRNA is mediated by SRRM3, and that SRRM3 is positioned downstream of RYBP.

In conclusion, in the present study, we focused on the role of RYBP in breast cancer. In addition to the findings related to
HCC and NSCLC showing that RYBP inhibits tumor progression, this study provides novel evidence that RYBP inhibits tumor growth and metastasis in breast cancer. Furthermore, our results suggest that SRRM3 and REST-003 lie downstream of RYBP. In future research, we will continue to explore the precise molecular mechanisms of RYBP, SRRM3, REST-003, and other potential molecules that may be involved in this process. We would also like to explore the epigenetic changes induced by overexpression of RYBP in breast cancer cells. Our findings support the notion that RYBP may offer a potential therapeutic target in breast cancer.

\section{Acknowledgements}

The present study was supported by the National Natural Science Foundation of China (81372821).

\section{References}

1. Stewart BW and Kleihues P: World cancer report. World Cancer Rep 45: 12-351, 2003.

2. Chen W, Zheng R, Baade PD, Zhang S, Zeng H, Bray F, Jemal A, $\mathrm{Yu}$ XQ and He J: Cancer statistics in China, 2015. CA Cancer J Clin 66: 115-132, 2016.

3. Siddiqui JA, Singh A, Chagtoo M, Singh N, Godbole MM and Chakravarti B: Phytochemicals for breast cancer therapy: Current status and future implications. Curr Cancer Drug Targets 15: 116-135, 2015.

4. García E, Marcos-Gutiérrez C, del Mar Lorente M, Moreno JC and Vidal M: RYBP, a new repressor protein that interacts with components of the mammalian Polycomb complex, and with the transcription factor YY1. EMBO J 18: 3404-3418, 1999.

5. Wang W, Qin JJ, Voruganti S, Nag S, Zhou J and Zhang R; Polycomb Group: Polycomb Group (PcG) proteins and human cancers: Multifaceted functions and therapeutic implications. Med Res Rev 35: 1220-1267, 2015.

6. Chen D, Zhang J, Li M, Rayburn ER, Wang H and Zhang R: RYBP stabilizes p53 by modulating MDM2. EMBO Rep 10: 166-172, 2009.

7. Sánchez-Beato M, Sánchez E, González-Carreró J, Morente M, Díez A, Sánchez-Verde L, Martín MC, Cigudosa JC, Vidal M and Piris MA: Variability in the expression of polycomb proteins in different normal and tumoral tissues. A pilot study using tissue microarrays. Mod Pathol 19: 684-694, 2006.

8. Wang W, Cheng J, Qin JJ, Voruganti S, Nag S, Fan J, Gao Q and Zhang R: RYBP expression is associated with better survival of patients with hepatocellular carcinoma (HCC) and responsiveness to chemotherapy of HCC cells in vitro and in vivo. Oncotarget 5: 11604-11619, 2014.

9. Novak RL and Phillips AC: Adenoviral-mediated Rybp expression promotes tumor cell-specific apoptosis. Cancer Gene Ther 15: 713-722, 2008.

10. Taylor BS, Schultz N, Hieronymus H, Gopalan A, Xiao Y, Carver BS, Arora VK, Kaushik P, Cerami E, Reva B, et al: Integrative genomic profiling of human prostate cancer. Cancer Cell 18: 11-22, 2010.

11. Lando M, Holden M, Bergersen LC, Svendsrud DH, Stokke T, Sundfør K, Glad IK, Kristensen GB and Lyng H: Gene dosage, expression, and ontology analysis identifies driver genes in the carcinogenesis and chemoradioresistance of cervical cancer. PLoS Genet 5: e1000719, 2009.

12. Voruganti S, Xu F, Qin JJ, Guo Y, Sarkar S, Gao M, Zheng Z, Wang MH, Zhou J, Qian B, et al: RYBP predicts survival of patients with non-small cell lung cancer and regulates tumor cell growth and the response to chemotherapy. Cancer Lett 369: 386-395, 2015

13. Lee NS, Evgrafov OV, Souaiaia T, Bonyad A, Herstein J, Lee JY, Kim J, Ning Y, Sixto M, Weitz AC, et al: Non-coding RNAs derived from an alternatively spliced REST transcript (REST-003) regulate breast cancer invasiveness. Sci Rep 5: 11207, 2015.

14. Franken NA, Rodermond HM, Stap J, Haveman J and van Bree C: Clonogenic assay of cells in vitro. Nat Protoc 1: 2315-2319, 2006. 
15. Kramer N, Walzl A, Unger C, Rosner M, Krupitza G, Hengstschläger $M$ and Dolznig $H$ : In vitro cell migration and invasion assays. Mutat Res 752: 10-24, 2013.

16. Lee YJ, Lin WL, Chen NF, Chuang SK and Tseng TH: Demethylwedelolactone derivatives inhibit invasive growth in vitro and lung metastasis of MDA-MB-231 breast cancer cells in nude mice. Eur J Med Chem 56: 361-367, 2012.

17. Suetsugu A, Honma K, Saji S, Moriwaki H, Ochiya T and Hoffman RM: Imaging exosome transfer from breast cancer cells to stroma at metastatic sites in orthotopic nude-mouse models. Adv Drug Deliv Rev 65: 383-390, 2013.

18. Sparmann A and van Lohuizen M: Polycomb silencers control cell fate, development and cancer. Nat Rev Cancer 6: 846-856, 2006.

19. Giacinti C, Giordano A and Giordano A: RB and cell cycle progression. Oncogene 25: 5220-5227, 2006.

20. Koliadi A, Nilsson C, Holmqvist M, Holmberg L, de La Torre M, Wärnberg F and Fjällskog ML: Cyclin B is an immunohistochemical proliferation marker which can predict for breast cancer death in low-risk node negative breast cancer. Acta Oncol 49: 816-820, 2010

21. Niméus-Malmström E, Koliadi A, Ahlin C, Holmqvist M, Holmberg L, Amini RM, Jirström K, Wärnberg F, Blomqvist C, Fernö M, et al: Cyclin B1 is a prognostic proliferation marker with a high reproducibility in a population-based lymph node negative breast cancer cohort. Int J Cancer 127: 961-967, 2010.
22. Foroni C, Broggini M, Generali D and Damia G: Epithelialmesenchymal transition and breast cancer: Role, molecular mechanisms and clinical impact. Cancer Treat Rev 38: 689-697, 2012.

23. Hanahan D and Weinberg RA: Hallmarks of cancer: The next generation. Cell 144: 646-674, 2011.

24. Palena C, Roselli M, Litzinger MT, Ferroni P, Costarelli L, Spila A, Cavaliere F, Huang B, Fernando RI, Hamilton DH, et al: Overexpression of the EMT driver brachyury in breast carcinomas: Association with poor prognosis. J Natl Cancer Inst 106: $106,2014$.

25. Perl AK, Wilgenbus P, Dahl U, Semb H and Christofori G: A causal role for E-cadherin in the transition from adenoma to carcinoma. Nature 392: 190-193, 1998.

26. Jiao W, Miyazaki K and Kitajima Y: Inverse correlation between E-cadherin and Snail expression in hepatocellular carcinoma cell lines in vitro and in vivo. Br J Cancer 86: 98-101, 2002.

27. Rosivatz E, Becker KF, Kremmer E, Schott C, Blechschmidt K, Höfler $\mathrm{H}$ and Sarbia M: Expression and nuclear localization of Snail, an E-cadherin repressor, in adenocarcinomas of the upper gastrointestinal tract. Virchows Arch 448: 277-287, 2006. 